\title{
Diacronie
}

Studi di Storia Contemporanea

$N^{\circ} 23,3$ | 2015

Mediterraneo cosmopolita

\section{Erica Ianiro, Levante. Veneti e Ottomani nel XVIII secolo}

Luca Zuccolo

\section{Q OpenEdition \\ Journals}

\section{Edizione digitale}

URL: http://journals.openedition.org/diacronie/2400

DOI: $10.4000 /$ diacronie. 2400

ISSN: 2038-0925

\section{Editore}

Association culturelle Diacronie

\section{Notizia bibliografica digitale}

Luca Zuccolo, "Erica Ianiro, Levante. Veneti e Ottomani nel XVIII secolo », Diacronie [Online], № 23, 3 | 2015, documento 5, Messo online il 29 octobre 2015, consultato il 23 septembre 2020. URL : http:// journals.openedition.org/diacronie/2400; DOI : https://doi.org/10.4000/diacronie.2400 
N. 23 | 3|2015 Mediterraneo cosmopolita: le relazioni culturali tra Turchia ed Europa

\title{
RECENSIONE:
}

\section{Erica IANIRO, Levante. Veneti e Ottomani nel XVIII secolo, Venezia, Marsilio, 2014, $415 \mathrm{pp}$.}

\author{
a cura di Luca ZUCCOLO *
}

Venezia e l'Impero ottomano hanno intessuto e intrattenuto rapporti costanti in ogni fase della loro storia come dimostrato in numerosi studi sia sulla città lagunare sia sull'Impero dei Turchi. Spesso in contrasto, questi due attori dominanti nella storia del Levante hanno condiviso esperienze simili più di qualsiasi altra realtà europea 0 mediterranea, al punto da creare, nei Balcani, una dipendenza veneziana dalla fiscalità ottomana come testimoniato dai documenti ottomani cinquecenteschi, dove maggiormente si può intravedere la stretta relazione tra i due Stati ${ }^{1}$.

All'interno di questo filone interpretativo ${ }^{2}$ che supera la teleologia delle visioni precedenti si pone anche il volume di Erica Ianiro, Levante. Veneti e Ottomani nel XVIII secolo, nel quale l'autrice, sfruttando le fonti venete presenti nell'Archivio di Stato del capoluogo lagunare e confrontandole con quelle ottomane conservate nel Başbakanlık Osmanlı Arşivi di Istanbul, propone una descrizione dei rapporti commerciali tra l'Impero e Venezia in alcune scale del Levante: Larnaca (Cipro); Aleppo; Smirne; e Salonicco.

\footnotetext{
${ }^{1}$ Questi documenti si possono trovare sia nell'Archivio di Stato veneto sia nel Başbakanlık Osmanlı Arşivi. Per una raccolta dei documenti veneziani si veda: GÖKBİLGIN, M. Tayyip, «Venedik Devlet Arşivindeki Vesikalar Külliyatında Kanunî Sultan Süleyman Devri Belgeleri», in Belgeler, I, 1964, pp. 119-220; ID., «Venedik Devlet Arşivindeki Türkçe Belgeler Kolleksiyonu ve Bizimle İlgili diğer Belgeler», in Belgeler, V-VIII, 1968-1971, pp. 1-151; PEDANI, Maria Pia, I "Documenti turchi" dell'Archivio di Stato di Venezia, inventario della miscellanea, Roma, Ministero per i Beni Culturali e Ambientali, 1994; ID., Le carte del sultano nell'Archivio di Stato di Venezia, in PEDANI, Maria Pia, BORTOLOZZO, Patrizia, Le carte del sultano nell'Archivio di Stato di Venezia. Catalogo della mostra documentaria, Venezia, Archivio di Stato di Venezia, 2010, pp. 15-20.

${ }^{2} \mathrm{Si}$ ricordano a questo proposito, solo per citarne alcune, l'opera di Daniel Panzac, quella di Donald Quataert e quella di Suraiya Faroqhi. Si vedano: PANZAC, Daniel, La peste dans l'Empire Ottoman: 170o-185o, Louvain, Editions Peeters, 1985; ID., La caravane maritime, Paris, CNRS Editions, 2004; QUATAERT, Donald, The Ottoman Empire 1700-1922, Cambridge, Cambridge University press, 2000; FAROQHI, Suraiya, The Venetian presence in the Ottoman Empire (1600-1630), in ISLAMOGLU-INAN, Huri (edited by), The Ottoman Empire and the World-Economy, New York, Cambridge University press, 2004, pp. 311-344.
} 
Lo studio, che si inserisce un panorama storiografico innovativo, sia a livello metodologico sia dal punto di vista delle fonti utilizzates, ha come primo obiettivo quello di mettere in discussione le visioni stereotipate, orientaliste e spesso acriticamente accettate che vogliono vedere nel XVIII secolo un periodo di estrema decadenza sia per la città lagunare sia per l'Impero. Come ricorda Vera Costantini nella prefazione:

La contrapposizione ideologica tra Ancien Régime e modernità non trova, infatti, corrispondenza nelle fonti settecentesche ottomane e veneziane, né, conseguentemente, in questo volume. Traspare piuttosto un concetto "esteso" di Levante 4 .

Sebbene durante il Settecento entrambe le realtà in oggetto siano andate incontro a rovesci militari, politici ed economici che videro, da un lato la fine della Repubblica di Venezia (1797) e, dall'altro, l'indebolimento dell'Impero ottomano in seguito alle riduzioni territoriali e ad una sempre maggiore influenza/interferenza europea, si devono registrare anche le dinamiche di riforma e di sviluppo socio-economico che interessarono queste due realtà mediterranee. Una delle finalità dell'autrice che così conclude il volume:

Ho voluto porre l'accento sulle fonti primarie archivistiche veneziane - e in modo secondario ottomane - per lo studio del Mediterraneo orientale nel XVIII secolo, un secolo spesso bollato di disinteresse e declino economico per Venezia e per il Mediterraneo ottomano. L'immagine che si ottiene [...] è estremamente variegata. In altre parole, non si riesce a tracciare una linea netta della realtà ma ci troviamo davanti a tanti colori e a tante sfumature: il Mediterraneo e la realtà veneziana erano molto più complessi di quelli descritti a lungo nella bibliografia secondaria, che spesso ha relegato in indistinto e grigio monocromo secondo piano la vivacità sociale, commerciale e diplomatica veneziana del Levante. La prima e più importante constatazione è l'inesistenza di un declino assoluto per Venezia [...], così non lo fu per gli ottomani. Al contrario, il XVIII secolo rappresentò per l'impero un periodo di grandi trasformazioni nei rapporti internazionali e sul piano interno5.

\footnotetext{
3 Filone storiografico di cui è esempio il volume di COSTANTINI, Vera, Il Sultano e l'isola contesa, Cipro tra eredità veneziana e potere ottomano, Torino, UTET, 2009.

4 COSTANTINI, Vera, Prefazione, in IANIRO, Erica, Levante. Veneti e Ottomani nel XVIII secolo, Venezia, Marsilio, 2014, p. 10.

5 IANIRO, Erica, Levante, cit., pp. 299-300.
} 
Un Levante "esteso" e mosaicato traspare dalle pagine di questo volume contrapposto al monolite fino a poco tempo addietro descritto dalla bibliografia secondaria scarsamente o per nulla a conoscenza delle fonti dirette ottomanoveneziane. Le stesse che rendono questa storia del Levante un interessante approccio comparativo e innovativo sia dal punto di vista tematico che metodologico. Erica Ianiro, infatti, grazie ai documenti del fondo Cinque Savi alla Mercanzia e alle fonti ottomane - Hatt-ı Hümâyun e Muallim Cevdet - ricostruisce non solo una storia economica degli scambi mercantili del Mediterraneo orientale, attraverso lo studio di quattro scali strategici per i commerci veneti, ma propone anche uno spaccato della vita sociale e dei mutamenti che durante tutto il secolo influenzarono le vite e le azioni dei veneziani e dei loro omologhi ottomani. Il mare e i porti, quindi, divengono il punto di partenza per un'analisi più profonda che partendo dalla struttura fiscale ottomana $\mathrm{e}$ dalla sua evoluzione - descritta e spiegata nel primo capitolo - si snoda attraverso le pagine del saggio, nell'esposizione dei principali prodotti commerciali scambiati negli scali di Larnaca, Aleppo, Smirne e Salonicco, per essere completata, poi, dalle peculiari relazioni sociali che si creano e si modificano durante tutto il secolo in questi porti e nelle società veneto-ottomane.

Non solo storia locale, o storia economica, ma anche storia sociale e dei mutamenti socio-politici che hanno modificato e condizionato lo sviluppo veneto-ottomano nel XVIII secolo. In questo saggio, infatti, si può osservare il ruolo chiave svolto dagli ayan ${ }^{6}$ all'interno dell'Impero ottomano e, allo stesso tempo, una loro riqualificazione in opposizione alla vulgata storiografica tradizionale che li ha sempre descritti in contrapposizione al potere centrale dei Sultani. Figure decisive nel Lale Devri (Età dei Tulipani), gli ayan hanno giocato un ruolo fondamentale nella storia ottomana, non solo a livello politico, ma anche sociale, così come i pirati lo hanno svolto nei confronti di Venezia e della sua politica mercantile che ha visto proprio nel XVIII secolo, ritorni il convoglio - ed evoluzioni.

Se il primo capitolo serve per inquadrare il XVIII secolo dal punto di vista politico sia per quanto riguarda l'Impero ottomano che la Repubblica di Venezia, nel difficile equilibrio internazionale dell'epoca illuminista, il secondo capitolo, al contrario, è

\footnotetext{
${ }^{6}$ Di estrazione sociale variegata gli ayan, come ricorda l'autrice, emersero dalle complesse realtà provinciali a partire dalla seconda metà del XVI secolo quando il controllo del potere centrale iniziò a ridursi accrescendo conseguentemente quello dei vali e dei sancakbeyler (uomini preposti al governo provinciale). L'atteggiamento sempre più dispotico di questi governatori costrinse un numero sempre maggiore di notabili nell'Anatolia e nei Balcani ad organizzarsi e a reagire al dispotismo per proteggere le popolazioni locali. L'emergere di queste figure, strettamente connesse al potere centrale in quanto mütesellim (funzionari statali per la riscossione delle tasse), per tanto permise ai Sultani un maggiore controllo del territorio e una più capillare relazione con le popolazioni locali.
} 
dedicato agli aspetti economici e in particolare al sistema fiscale ottomano e all'istituzione veneziana dei consolati: due istituzioni che daranno la cifra dei rapporti veneto-ottomani e dello sviluppo di entrambi i settori commerciali. Commerci che vengono descritti nella terza parte di questo capitolo, attraverso lo studio dei principali prodotti scambiati nelle scale del Levante.

I capitoli dal terzo al sesto, vero nucleo del saggio, propongono, invece, un cambiamento nella scala di grandezza, passando dalla dimensione euro-mediterranea a quella locale. Ogni capitolo è dedicato a un porto, a partire da Larnaca nell'isola di Cipro. In questi quattro capitoli le fonti e i documenti d'archivio parlano direttamente al lettore in quale viene proiettato nella dimensione strategica per le reti venetoottomane nel Levante di questi quattro porti e delle loro peculiari reti socio-politiche.

Di Larnaca vengono descritte l'amministrazione ottomana e quella consolare veneziana, inserite in un sistema reticolare di relazioni sociali descritte nelle fonti venete attraverso contratti matrimoniali, affitti e vendite, ma anche attraverso gli interessi commerciali legati alla produzione vinicola.

Con Aleppo si viene catapultati dalle reti marittime a quelle terrestri. Centro fondamentale per le vie carovaniere arabe, mesopotamiche e anatoliche, che fanno della città siriana un punto focale degli scambi imperiali, Aleppo, al pari di Larnaca, si propone come un emporio commerciale strategico per la Repubblica di Venezia, non solo per i grandi commerci provenienti da Oriente, ma anche per gli scambi a più breve raggio, che i mercanti veneziani possono svolgere nei porti aleppini sulla costa mediterranea: Alessandretta; Latachia; Tripoli; Acri e Giaffa. Qui, a differenza di Larnaca, si inizia a intravedere il vasto mosaico di popoli e tradizioni con cui la colonia veneta si trova a condividere spazi e commerci.

Mosaico di popoli ben più visibile e rilevante negli ultimi due scali descritti dall'autrice: Smirne e Salonicco. In entrambi i casi si percepisce l'avvicinarsi al centro dell'Impero sia dal punto di vista amministrativo che sociale. Maggiore diviene il peso delle comunità ebraiche e greche, che modificano e influenzano pesantemente la dinamica commerciale ed economica degli interessi veneti. Attraverso i rapporti consolari, infatti, si ottiene un chiaro spaccato della vita sociale e commerciale di questi due porti e delle loro criticità, dovute sia a cause naturali (terremoti e pestilenze a Smirne), sia a cause sociali (i giannizzeri a Salonicco). Sullo "sfondo" le materie prime e i prodotti del commercio internazionale: tabacco, vino, panni lana, cotone, granaglie, vetrerie e carta.

Reti relazionali, reti politiche, reti commerciali, reti personali: questo è il poliedrico panorama che emerge dalle fonti nella descrizione del Levante veneto-ottomano 
proposta da Erica Ianiro. Un Levante "esteso", come sottolinea Vera Costantini, e in continuo movimento; tutt'altro che fermi e decadenti, il Levante ottomano e, conseguentemente, Venezia, si propongono come due dei motori dell'evoluzione di una regione che sarà al centro della dinamica euro-mediterranea e globale nel secolo successivo. L'apporto di questo volume alla storiografia lagunare e ottomana è dunque di prim'ordine e fondamentale per le prospettive analitiche che apre, a partire da quelle economiche, ma ancor di più in riferimento a quelle sociali e umane. Le fonti, infatti, descrivono una società vivace che delinea diversi aspetti della vita e delle interrelazioni economiche che interessano famiglie e mercanti veneti, espressione sia della società lagunare che di quella levantina, di cui fanno parte ricoprendo un ruolo privilegiato. I veneziani, infatti, al pari dei genovesi, hanno dato origine alla comunità levantina ottomana infondendole i loro valori e caratterizzandola dal punto di vista socioculturale.

Il saggio, oltre a presentare un'analisi e una metodologia significative per lo studio della regione si completa con una ricca appendice quantitativa in cui pratiche tabelle elencano e approfondiscono quanto descritto nel testo fornendo utili dati sui commerci e sulle merci scambiate nelle quattro scale di riferimento. Altrettanto utili e approfonditi sono il glossario e la ricca bibliografia che completa egregiamente il vasto apparato di fonti utilizzate dall'autrice.

Fondamentale per chi voglia studiare il Levante veneto-ottomano e l'economia settecentesca, il saggio qui presentato è comunque adatto a un vasto pubblico per le suggestioni e le informazioni fornite dall'autrice attraverso una chiara descrizione dei fatti e delle dinamiche relazionali tra Venezia e Impero ottomano. 


\section{* L'autore}

Dopo aver conseguito la laurea in Storia Contemporanea e il titolo di dottore magistrale in Storia d'Europa presso l'Università di Bologna (2005 e 2008) ha ottenuto il titolo di Dottore di ricerca in Storia Contemporanea presso il SUM - Istituto Italiano di Scienze Umane - Napoli. I suoi campi di ricerca sono: la modernizzazione dell'Impero Ottomano (XIX-XX secolo), il ruolo della stampa ottomana nel contesto imperiale ed europeo e i movimenti sociali che hanno preparato l'avvento della società turca contemporanea.

URL: < http://www.studistorici.com/2010/12/o1/luca-zuccolo/ >

\section{Per citare questo articolo:}

ZUCCOLO, Luca, «Recensione: Erica IANIRO, Levante. Veneti e Ottomani nel XVIII secolo, Venezia, Marsilio, 2014, 415 pp.», Diacronie. Studi di Storia Contemporanea : Mediterraneo cosmopolita: Ie relazioni culturali tra Turchia ed Europa, 29/09/2015,

URL:< http://www.studistorici.com/2015/09/29/zuccolo_numero_23/ >

Diacronie Studi di Storia Contemporanea $\beta$ www.diacronie.it

Risorsa digitale indipendente a carattere storiografico. Uscita trimestrale. redazione.diacronie@hotmail.it

Comitato di redazione: Jacopo Bassi - Luca Bufarale - Elisa Grandi - Deborah Paci - Fausto Pietrancosta - Matteo Tomasoni - Luca Zuccolo

Diritti: gli articoli di Diacronie. Studi di Storia Contemporanea sono pubblicati sotto licenza Creative Commons 2.5. Possono essere riprodotti a patto di non modificarne i contenuti e di non usarli per fini commerciali. La citazione di estratti è comunque sempre autorizzata, nei limiti previsti dalla legge. 